> Chaque étape du cycle viral du VIH-l s'accompagne d'une modification de la morphologie et/ou des propriétés mécaniques de la particule entière ou de la capside virale. Le microscope à force atomique (AFM) est un outil physique extrêmement performant pour caractériser ces changements à l'échelle d'un virus unique. En effet, l'AFM permet, d'une part, la visualisation de capsides virales dans un environnement physiologique contrôlé, et, d'autre part, de sonder leurs propriétés mécaniques par nano-indentation. Enfin, la spectroscopie de force AFM peut permettre de caractériser les affinités entre les protéines d'enveloppe virale et les récepteurs cellulaires à l'échelle de la molécule unique. <

Le virus de l'immunodéficience humaine de type 1 (VIH-1) est un virus enveloppé à ARN positif simple brin appartenant à la famille des rétrovirus. Ce virus présente une capside virale conique protégeant le génome d'ARN viral, elle-même entourée de la matrice virale et d'une bicouche lipidique qui dérive de la cellule hôte (Figure 1). Depuis la découverte du VIH-1 en 1983 [1], de nombreuses études ont été réalisées, non seulement pour comprendre son cycle infectieux, mais aussi pour caractériser les particules virales. En particulier, la cristallographie par rayons $X$ et la résonance magnétique nucléaire (RMN) ont été utilisées pour obtenir la structure des protéines du VIH-1 [2-4], et des images obtenues par cryomicroscopie électronique (cryo$\varepsilon M$ ) ont permis de caractériser précisément la forme conique particulière de la capside $[5,6]$.

Récemment, l'utilisation d'outils issus de la physique a conduit à de nouvelles informations sur la dynamique d'assemblage du virus [7] ou sur son trafic dans la cellule [8]. Depuis une dizaine d'années, le recours à des microscopes de très haute résolution, comme le microscope à force atomique (AFM), a ouvert de nouvelles voies pour l'étude morphologique, mais aussi pour l'étude de la robustesse mécanique des virus [9, 10].

\section{Microscopie à force atomique pour l'étude du cycle viral}

\section{L'exemple du VIH-1}

Julien Bernaud ${ }^{1}$, Martin Castelnovo', Delphine Muriaux², Cendrine Faivre-Moskalenko ${ }^{1}$

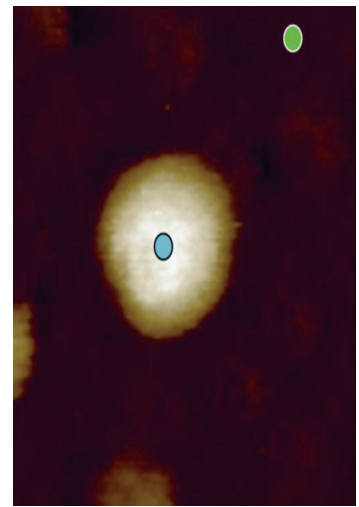

En effet, les conditions d'imagerie de l'AFM, comme celles de la cryo$\varepsilon M$, permettent de conserver la structure des échantillons analysés. De plus, même si l'AFM n'atteint pas une meilleure résolution que la cryo-EM, elle est la seule qui permette de caractériser les propriétés mécaniques de l'objet observé. Ainsi, l'AFM constitue un outil privilégié pour l'étude des propriétés physiques du VIH-l dans différentes phases de son cycle viral (Figure 1), de même que pour la comparaison des différentes propriétés physiques d'une famille virale à une autre. Le cycle de réplication du VIH-l (Figure 1) peut être schématisé de la façon suivante : la phase précoce de l'infection débute par la reconnaissance des glycoprotéines d'enveloppe du VIH par les récepteurs cellulaires CD4 et CXCR4 ou CCR5, qui permettent l'entrée de la capside virale par fusion de la membrane du virus avec celle de la cellule hôte. Puis, le génome viral ARN est rétrotranscrit en ADN proviral, libéré dans le cytosol et adressé au noyau dans un complexe préintégratif. Une fois dans le noyau, l'ADN est intégré dans le génome de la cellule hôte [11]. La phase tardive du cycle comprend la transcription du génome viral, l'épissage du transcrit primaire, la traduction des protéines virales, l'assemblage de la polyprotéine Gag (et Gag-Pol), de I'ARN génomique viral et des glycoprotéines d'enveloppe à la membrane de la cellule hôte [12], et, enfin, le bourgeonnement du virus [7]. Lors de sa sortie de la cellule hôte, le virus subit une étape de maturation au cours de laquelle sa morphologie change lors du clivage par la protéase virale de la polyprotéine Gag en protéines MA (matrice), CA (capside), NC (nucléocapside), p6, et deux peptides spl et sp2. 

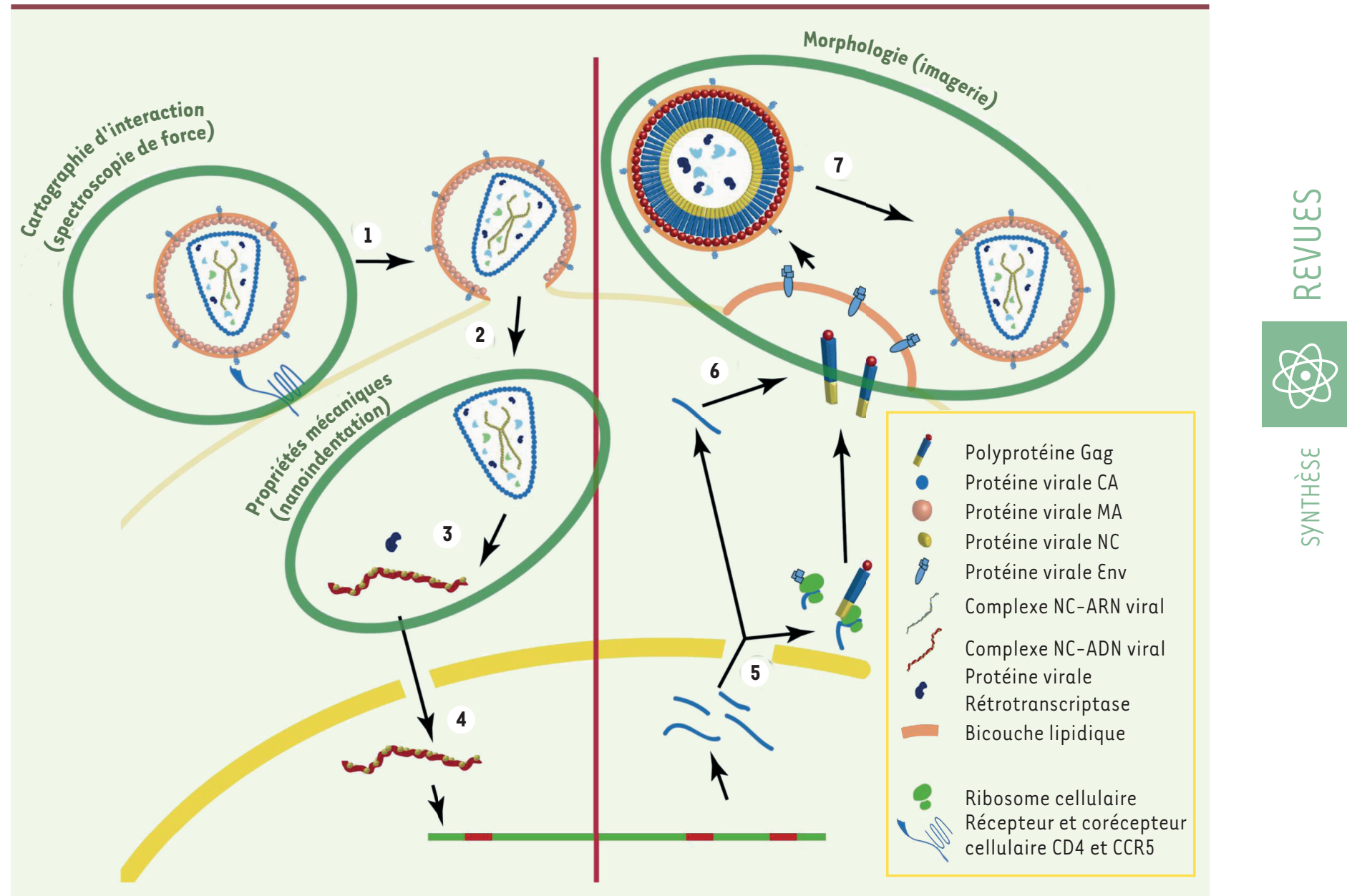

Figure 1. Cycle viral simplifié du VIH-1. L'étape de fixation (1) du virus sur le récepteur cellulaire (CD4/CCR5 par exemple) peut être étudiée en fonctionnalisant la pointe de l'AFM avec l'une des deux protéines (par spectroscopie de force). Après fusion des membranes virale et cellulaire, la capside est libérée dans le cytoplasme (2). La rétrotranscription et la décapsidation (3) conduisent à une modification importante des propriétés mécaniques qui peuvent être mesurées par nano-indentation AFM. L'intégration du génome dans l'ADN de la cellule (4) est suivie des étapes de transcription et de traduction des protéines virales (5). Les protéines virales s'assemblent à la membrane plasmique au site d'assemblage, étape suivie de celle de bourgeonnement du virus (6) et de maturation (7), au cours de laquelle la protéase virale clive la polyprotéine Gag en MA, CA, sp2, NC, spl et p6. La morphologie des particules virales produites par la cellule, ainsi que les modifications structurales liées à l'étape de maturation, peuvent être étudiées en exploitant le mode imagerie de l'AFM.

L’AFM, qui a été développée dans les années 1980 [13] pour la caractérisation physique des matériaux durs à l'échelle nanométrique, est de nos jours fréquemment utilisée pour la visualisation d'objets biologiques à l'échelle de la molécule unique [14-16]. L'un des avantages de l'AFM est de pouvoir visualiser avec une haute résolution spatiale les objets biologiques immergés dans un environnement physiologique contrôlé (milieu liquide, température, pH, etc.) [15].

L'AFM consiste à utiliser une pointe nanométrique montée sur un levier flexible pour mesurer la topographie d'une surface (Figure 2A). Son fonctionnement repose sur l'existence de forces présentes à très courte distance (échelle nanométrique) entre la pointe et la surface, et qui entraînent une déflexion du levier. Lors du balayage de la surface par la pointe, la position verticale de l'échantillon peut être ajus- tée, à l'aide d'une boucle de rétroaction, pour maintenir la déflexion (donc la force d'interaction) constante. Ces variations de position permettent de déterminer la forme des objets déposés sur la surface avec une résolution comparable aux dimensions de la pointe (2 à $10 \mathrm{~nm}$ ), comme cela est illustré sur la figure $2 B$, où l'on voit l'image typique d'une capside et d'une pseudoparticule virales (VLP) du VIH-l obtenue par AFM dans un environnement liquide. La couleur de l'image code ici pour la hauteur de l'objet, comme le montre la représentation graphique de la hauteur mesurée le long de la ligne de section indiquée. L'information obtenue est quantitative et permet la caractérisation de la 
A

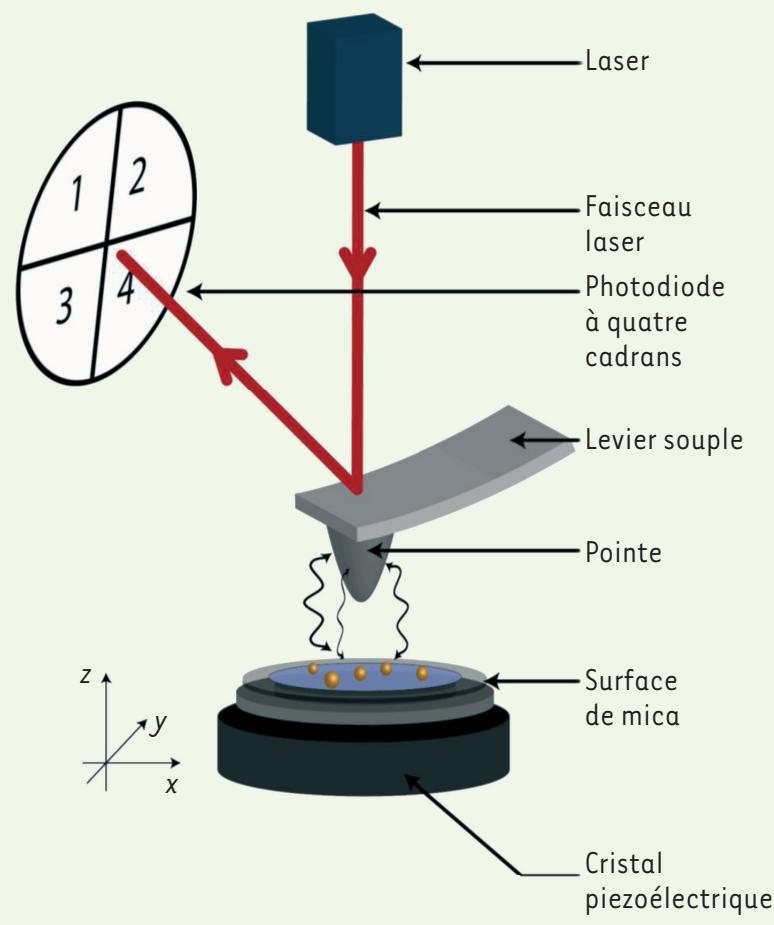

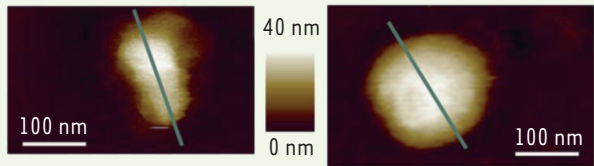
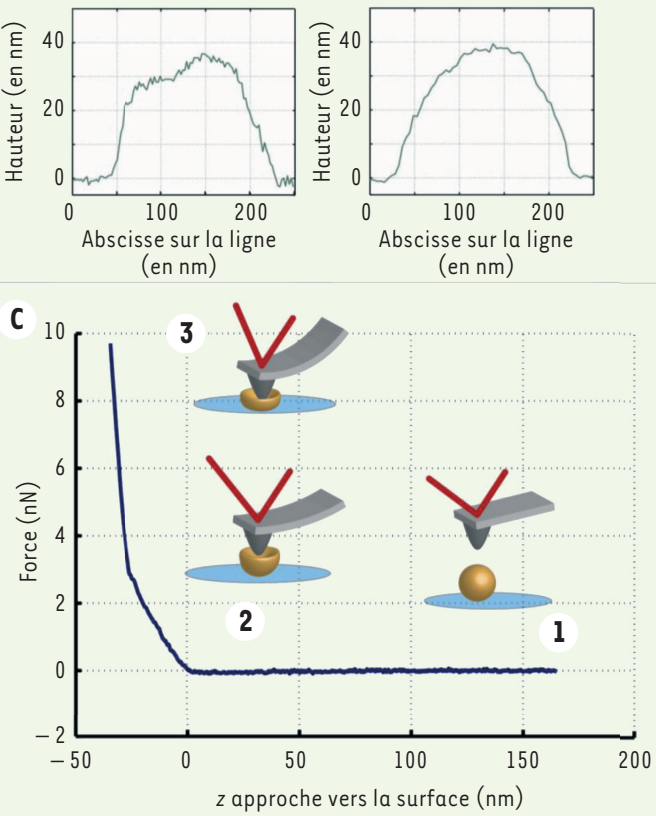

Figure 2. Utilisation de la microscopie à force atomique : principe et application à l'étude du VIH-1. A. Description du microscope à force atomique (AFM). La pointe (hauteur : $10 \mu \mathrm{m}$, diamètre à l'extrémité : $5 \mathrm{~nm}$ ), montée sur un levier souple, est en interaction avec la surface. Sous l'effet d'une différence de tension électrique, le cristal piézo-électrique peut se contracter ou se dilater, permettant le déplacement de la surface se trouvant dessus, sur des distances micrométriques. La présence d'objets (particules virales) sur celle-ci modifie l'interaction pointe-surface, entraînant une déflexion du levier, mesurée par la réflexion d'un laser vers une photodiode à quatre cadrans. II est ainsi possible de mesurer la topographie de la surface dans l'air ou dans un milieu physiologique. B. Mode imagerie. Image obtenue en milieu liquide (en haut) de la topographie d'une capside VIH-1 (à gauche) et d'une VLP VIH-1 (à droite) déposées sur une surface de mica fonctionnalisée par la polylysine. La couleur représente la hauteur comme on peut le voir sur les profils le long des sections indiquées. C. Mode indentation. II n'y a plus de balayage dans les directions $x$ et $y$, la pointe s'approche verticalement de la surface (au-dessus d'un virus, direction z). Lorsqu'il n'y a pas d'interaction, le levier est immobile (étape 1), sa déflexion est nulle. Au moment où la pointe entre en interaction avec l'objet, le levier et l'objet se déforment (étape 2). Par la suite, lorsque la déformation de l'objet est trop importante, seul le levier peut encore se déformer (étape 3). La force exercée par le levier est directement proportionnelle à sa raideur (connue) et à sa déflexion (mesurée).

morphologie des particules virales (taille, forme, asymétrie) grâce au mode imagerie de l'AFM (Figure 2B).

Outre ses performances pour l'observation, l'AFM permet aussi d'étudier les propriétés mécaniques locales d'objets déformables comme les virus. En positionnant la pointe à la verticale du virus, on peut ainsi appuyer sur la capside (nano-indentation, Figure 2C) et en mesurer la réponse à une déformation imposée de quelques nanomètres $[17,33](\rightarrow)$. La forme de la courbe ainsi obtenue est caractéristique de la raideur du virus. Ainsi, les propriétés mécaniques de la capside virale peuvent être quantifiées par nano- $(\rightarrow)$ Voir aussi pour exemple la Nouvelle de A. Kabla et K. Chalut, $m / s n^{\circ} 10$, octobre 2014, page 1061 indentation AFM afin de déterminer sa robustesse et, par exemple, relier cette robustesse à l'étape de déstabilisation virale, ou, de manière plus générale, au pouvoir infectieux du virus [18].
Les forces d'adhésion (non spécifiques ou lors d'une liaison ligand-récepteur) entre la pointe AFM et la surface peuvent également être quantifiées. En couplant cette information avec le balayage de la pointe, on obtient une cartographie de l'affinité pointeéchantillon (Figure 3A) : c'est ce que l'on appelle la spectroscopie dynamique de force AFM [19-21]. Par exemple, en fixant sur la pointe de l'AFM des protéines virales d'enveloppe du VIH-1, la spectroscopie de force AFM permet une mesure localisée des interactions de ces protéines virales avec les récepteurs présents dans la membrane externe d'une cellule immobilisée sur une surface [22], mimant ainsi l'interaction virus-cellule (Figure $3 B$ ). 
A
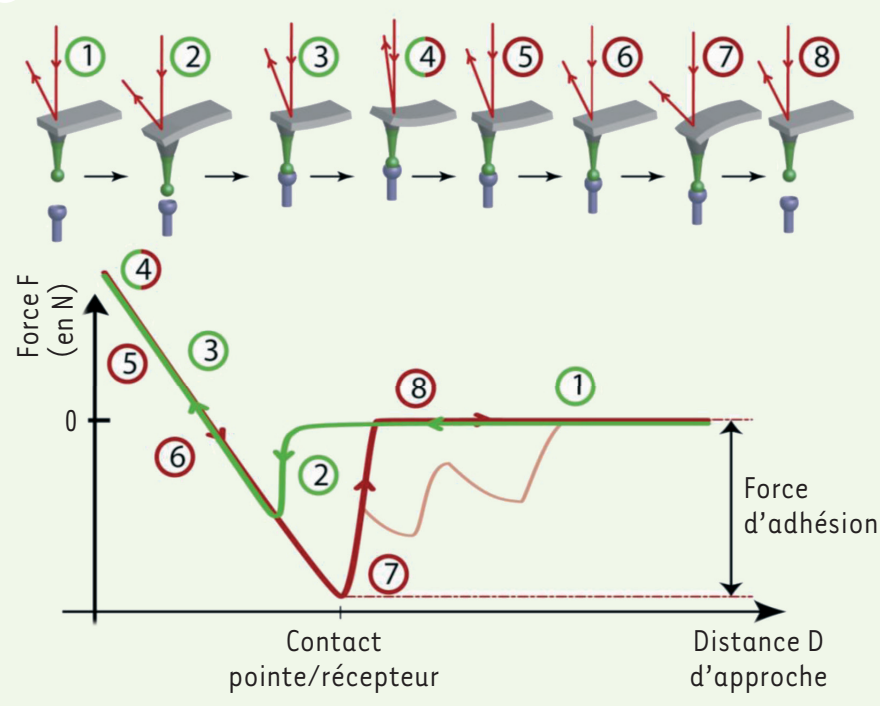

B
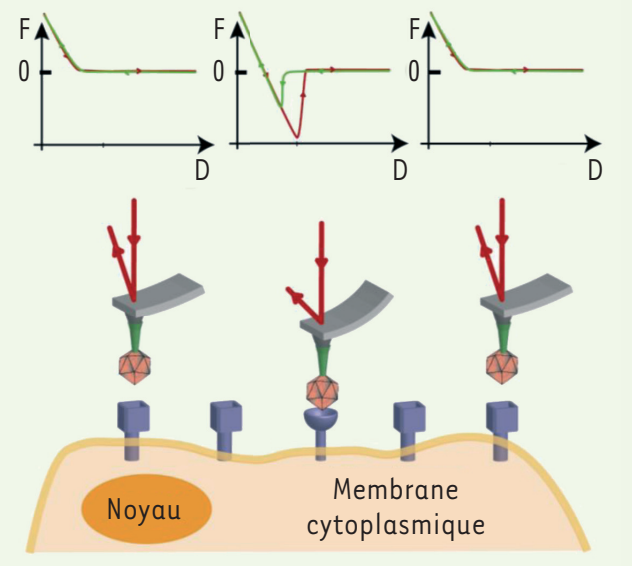

Figure 3. Spectroscopie de force AFM. A. Illustration schématique (en haut) et signal associé (en bas) de l'étude d'interaction d'un couple récepteur-protéines virales de surface. L'approche de la pointe fonctionnalisée (1) vers le récepteur augmentera l'interaction, ce qui occasionne une déflexion du levier (2) lors du contact, puis une déformation continue du levier (3) jusqu'à une valeur limite de consigne (4). Lors de l'éloignement de la pointe, l'interaction antigène-anticorps dominera (5) jusqu'à ce que la force de rappel du levier soit supérieure à l'attachement pointe/ récepteur (6), ce qui cassera la liaison antigène-anticorps et libérera le levier. La force maximale correspond à l'interaction entre le récepteur et l'antigène. Lorsque plusieurs récepteurs ou protéines de surface sont mis à contribution, le détachement de chaque récepteur peut être visible sur la courbe de force (courbe rouge claire). B. Principe simplifié de la spectroscopie de force. Un signal spécifique d'interaction est présent dans la courbe de force lorsque le récepteur correspond à celui du virus.

Dans ce qui suit, nous présenterons les travaux que nous avons réalisés par AFM pour caractériser certaines étapes du cycle viral du VIH-l mentionnées précédemment. $\varepsilon$ n conclusion, nous indiquerons des travaux similaires réalisés sur d'autres virus.

\section{Morphologie : observation de virus à l'échelle de la particule unique}

L'équipe de McPherson, qui a réalisé par AFM les premières images de lymphocytes infectés par le VIH-l ainsi que de particules VIH-l fixées sur une surface de verre [9], a révélé la grande variabilité de taille et de forme des particules virales produites par les cellules infectées.

Nous avons étudié la morphologie de pseudo-particules virales (VLP)l matures et de capsides purifiées du VIH-1 déposées sur une surface de mica fonctionnalisée, sans fixateur [23]. Cela permet, en combinant la visualisation de plusieurs centaines d'objets avec l'analyse quantitative des images obtenues, de mesurer de manière statistique les propriétés morphologiques d'une population de capsides virales. Grâce à un programme de traitement d'images, il est ainsi possible d'extraire, pour chaque particule virale examinée, sa carte d'iso-hauteur (ou

\footnotetext{
${ }^{1}$ Les VLP sont des nanoparticules qui ne contiennent pas l'intégrité du matériel génétique du virus (pseudo-virions). Ces particules non infectieuses contiennent des protéines virales et leur morphologie est presque identique à celle des virions.
}

courbes de niveau, Figure $4 \mathrm{~A}$ ) et ses caractéristiques morphologiques : hauteur maximale, grand axe et petit axe de l'objet asymétrique, diamètre équivalent d'un disque de même aire ou asymétrie. Les distributions corrélées de petit et grand axe d'une population de virus (Figure 4B) permettent ainsi de visualiser directement la taille la plus probable des particules virales, la dispersion de ces tailles (donnée par l'étendue de la distribution 2D), ainsi que leur asymétrie (proximité avec l'axe pointillé pour lequel les deux diamètres sont égaux). Comme le montre la figure $4 B$, le $\mathrm{VIH}-1$, rétrovirus enveloppé, présente une variabilité de taille beaucoup plus importante que le virus AAV (adenoassociated virus), virus à ADN non enveloppé à symétrie icosaédrique. De plus, les VLP du VIH - l sont isotropes, et leur distribution s'étale le long de l'axe pointillé de symétrie, alors que la distribution de taille des capsides du VIH-l s'éloigne de l'axe pointillé, en accord avec leur forme conique.

Des observations morphologiques plus fines ont pu être mises en évidence, grâce à la haute résolution de l'AFM, en comparant la morphologie de VLP matures du VIH-I assemblées en présence ou en l'absence d'un ARN viral pouvant être encapsidé (ARN $\psi$ ) [23]. L'ARN $\psi$ est 

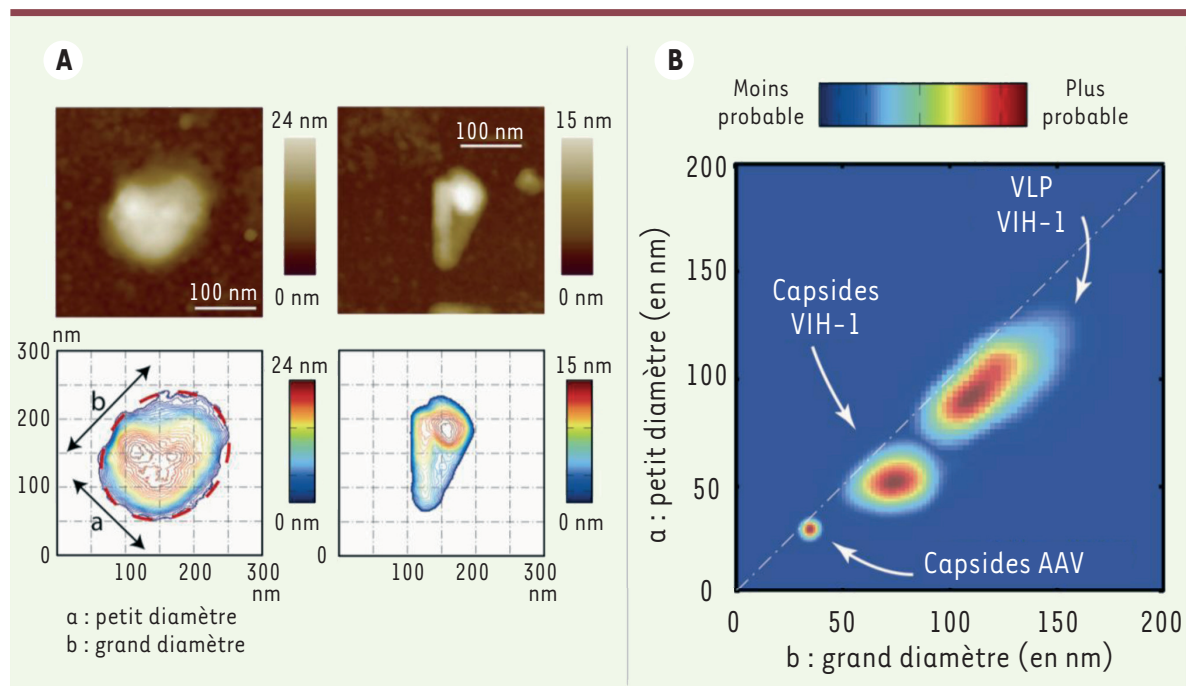
C - VLP VIH- $1-\psi(\mathrm{N}=248)$ - VLP VIH- $1+\psi(\mathrm{N}=211)$

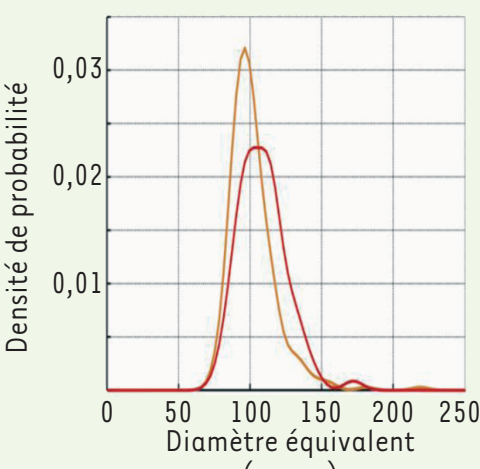

(en $\mathrm{nm}$ )

Figure 4. Imagerie AFM des pseudo-particules virales (VLP) et capsides du VIH-1. A. Topographie (en haut) et représentation iso-hauteur (en bas) d'une pseudo-particule virale (à gauche) et d'une capside (à droite) du VIH-l. Une analyse automatique permet de tirer certaines propriétés morphologiques comme la hauteur ou les dimensions caractéristiques (petit et grand diamètres a et b). B. Carte de densité de probabilité corrélée du petit diamètre et du grand diamètre pour trois populations de virus différents (capsides AAV, capsides du VIH-1 et VLP du VIH-1). Plus la zone est rouge, plus il est probable d'observer une particule avec ces paramètres morphologiques. C. Densité de probabilité du diamètre équivalent pour des particules du VIH-l assemblées en présence (orange), et en l'absence (rouge), d'ARN pseudo-viral contenant le signal d'encapsidation viral $\psi$.

un catalyseur de l'assemblage viral (Figure 1) [34]. Nous avons pu observer que les VLP contenant l'ARN viral $\psi$ ont statistiquement une taille inférieure à celle des VLP qui ont été assemblées autour d'ARN cellulaires, et que leur polydispersité est plus faible (Figure 4C). Ces résultats suggèrent que le signal $\psi$ exerce ainsi un rôle structurant pour les particules bourgeonnantes immatures, qui persiste après maturation du virus.

\section{Propriétés mécaniques : nano-indentation}

L'utilisation du levier comme sonde de force pour indenter les virus s'est révélée une technique privilégiée pour mesurer leurs propriétés mécaniques. Les courbes de force (Figure 5) permettent d'extraire la déformation (indentation) du virus au cours de l'approche, à condition de connaître le comportement du levier en l'absence du virus, c'est-àdire de pouvoir mesurer sa raideur par une expérience indépendante. Le levier AFM est mécaniquement équivalent à un ressort de raideur $k_{\text {lev }}$. La force, produit de $k_{\text {lev }}$ par la déflexion du levier, est alors une fonction linéaire du déplacement lorsque le levier indente une surface dure (comme une surface de mica, voir Figure 5B, en vert).

Si l'indentation est réalisée sur une capside virale, celle-ci se déforme. Le signal mesuré est la combinaison de cette déformation et de la déflexion du levier comme par exemple, pour une capside VIH-l (Figure 5B, en orange) dont la courbe de force présente une pente différente de celle qui est observée si l'indentation est réalisée sur une surface dure de mica (Figure 5B, en vert). Lorsque l'on appuie sur une VLP du VIH-l (Figure 5B, en bleu), on observe d'abord un régime linéaire de pente très faible, qui correspond à la déformation de l'enveloppe lipidique de la particule, puis un second régime linéaire de même pente que celui qui est observé pour la déformation de la capside seule du VIH, suggérant que dans ces conditions, on déforme la capside à l'intérieur de la particule enveloppée.

Pour quantifier ces différents comportements, on applique généralement un modèle d'élasticité linéaire, c'est-à-dire que le virus et le levier se comportent comme deux ressorts en série. La pente mesurée $k_{\text {mes }}$ est alors reliée à la raideur du virus $k_{\text {vir }}$ par la relation suivante :

$$
k_{\text {vir }}=\frac{k_{\text {lev }} \cdot k_{\text {mes }}}{k_{\text {lev }}-k_{\text {mes }}}
$$

Plus la constante $k_{\text {vir }}$ sera importante, plus la capside pourra être considérée comme rigide. Nous obtenons les valeurs suivantes pour la capside du VIH : $k_{\text {cap }} \sim 1,5 \mathrm{~N} / \mathrm{m}$, et, pour l'enveloppe de la particule (bicouche lipidique) : $k_{e n v} \sim 0,12 \mathrm{~N} / \mathrm{m}$, soit une rigidité environ 10 fois moindre pour l'enveloppe que pour la capside protéique.

De nombreux virus ont été caractérisés par cette méthode [24]: les raideurs mesurées vont de quelques centièmes de $\mathrm{N} / \mathrm{m}$ à quelques $\mathrm{N} / \mathrm{m}$. À noter que dans certains cas, on peut aller jusqu'à observer la rupture de la capside virale [17].

Par cette méthode, il est possible de suivre l'évolution des propriétés mécaniques de la particule au cours du cycle viral en utilisant des virus mutés. Ainsi, l'étape de maturation, durant laquelle la protéase virale clive la 


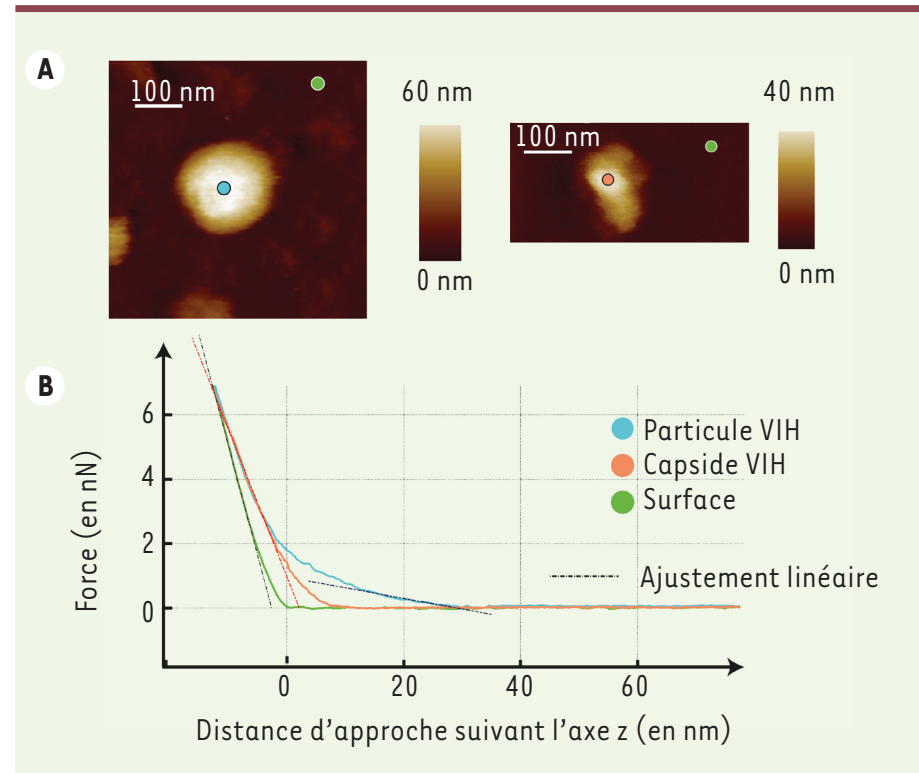

Figure 5. Expérience de nano-mécanique. A. Image dans un environnement liquide (tampon physiologique) d'une VLP du VIH-I (à gauche) et d'une capside du VIH-1 (à droite). Les pastilles représentent les points de contact avec la pointe de l'AFM lors des expériences de nano-indentation. B. Courbes de force en différents points réalisées avec un levier de raideur $0,6 \mathrm{~N} / \mathrm{m}$ : au centre la particule du VIH-1, en bleu, sur une capside du VIH-l, en orange, et sur la surface de mica, en vert. Si la courbe de force évolue de manière affine, la déformation de la particule peut se modéliser par la déformation linéaire d'un simple ressort. La mesure de la pente d'un ajustement linéaire (trait en pointillé) permet d'extraire la raideur de la particule.

polyprotéine Gag en plusieurs protéines, s'accompagne d'une réorganisation de la capside virale couplée à une diminution d'un facteur 10 de la raideur mesurée pour des VLP du VIH-1 [18]. De même, l'équipe de Rousso a pu mettre en évidence que la concentration accrue en protéines d'enveloppe de particules immatures entraîne une augmentation de la rigidité des particules, accompagnée d'une diminution de leur capacité d'entrer dans les cellules [25], corrélant ainsi une propriété mécanique de la capside à une propriété biologique du virus (pouvoir infectieux).

\section{Cartographie d'interactions : la spectroscopie de force AFM}

La pointe est sensible à tout objet se trouvant à la surface de l'échantillon. En couplant biochimiquement la pointe avec des molécules, des protéines ou même des virus, il est possible d'accentuer certaines interactions et de cartographier les affinités des objets se trouvant sur la surface avec les structures associées à la pointe $[35](\rightarrow)$.

$(\rightarrow)$ Voir pour exemple la Nouvelle de $\mathrm{S}$. Kasas et al., $\mathrm{m} / \mathrm{s} \mathrm{n}^{\circ} 4$, avril 2015, page 369

Par exemple, en associant une protéine d'enveloppe virale à la pointe, on peut mesurer la force maximale d'adhésion entre celle-ci et un récepteur fixé sur la surface (Figure $3 A$ ). Cette méthode permet ainsi de mieux comprendre quel récepteur est privilégié lors de la recon- naissance du virus par la cellule. Elle a été utilisée pour caractériser les récepteurs cellulaires de la protéine d'enveloppe HA (hémagglutinine) du virus de la grippe [26]. Le temps nécessaire à la libération de la protéine par le récepteur, mesuré en observant temporellement le signal pour une force de retrait du levier donnée (Figure 3B), permet la détermination de la constante cinétique de désorption. Cette dernière mesure a été réalisée par l'équipe de Wirtz [22] pour le virus VIH-1 lié à son récepteur cellulaire CD4 et aux corécepteurs CCR5 et CXCR4.

\section{Conclusion}

À ce jour, outre les études concernant le VIH-l, des études d'imagerie par AFM ont été réalisées sur plusieurs types de virus comme les bactériophages [27], des parvovirus [28], ou des virus plus complexes, comme le HSV (virus de l'herpès simplex) [29]. Comme en cryo-EM, la résolution de cette méthode est telle qu'il est possible d'observer les protubérances des protéines de structure de certains virus non enveloppés qui sont en général à symétrie icosaédrique, et d'en déterminer leur nombre de triangulation ainsi que leurs axes de symétrie [30]. En couplant la haute résolution pour l'imagerie et les expériences de nano-indentation, I'AFM permet de déterminer des effets subtils comme le rôle mécanique du génome présent au sein de la capside. Des expériences de nano-indentation AFM réalisées sur des bactériophages remplis d'ADN double brin de différentes longueurs ont ainsi permis de mettre en évidence l'existence d'une pression interne exercée par le génome sur la paroi de la capside [31] (voir aussi [33]). Par ailleurs, dans le cas du virus minute des souris (MVM), un virus à symétrie icosaédrique, des différences de raideur de la capside virale ont pu être mesurées selon le positionnement du virus sur une surface (sur une face, une arrête ou un sommet) et il a été possible de les relier aux points d'attache de l'ADN à l'intérieur de la capside [28].

L'AFM permet ainsi d'apporter des informations physiques quantitatives à l'échelle de la particule unique, et d'éclairer certaines propriétés biologiques au cours du cycle infectieux. Son avantage par rapport à la microscopie électronique est de permettre de coupler une mesure mécanique à l'imagerie haute résolution, offrant un outil précieux pour l'étude des propriétés physiques des particules analysées, comme les virus. De plus, le contrôle de l'environnement de l'échantillon étudié par AFM, permet d'envisager l'étude des comportements hors équilibre [32], puisqu'il est possible de modifier in situ la force ionique (en ajoutant des ions), 
le $\mathrm{pH}$ (en modifiant la nature du tampon) ou la pression osmotique auxquels sont exposées les capsides virales. $\diamond$

\section{SUMMARY}

Atomic force microscopy: a tool to analyze the viral cycle

Each step of the HIV-1 life cycle frequently involves a change in the morphology and/or mechanical properties of the viral particle or core. The atomic force microscope (AFM) constitutes a powerful tool for characterizing these physical changes at the scale of a single virus. Indeed, AFM enables the visualization of viral capsids in a controlled physiological environment and to probe their mechanical properties by nano-indentation. Finally, AFM force spectroscopy allows to characterize the affinities between viral envelope proteins and cell receptors at the single molecule level. $\diamond$

\section{LIENS D'INTÉRÊT}

Les auteurs déclarent n'avoir aucun lien d'intérêt concernant les données publiées dans cet article.

\section{RÉFÉRENCES}

1. Barré-Sinoussi F, Chermann JC, Rey F, et al. Isolation of a T-lymphotropic retrovirus from a patient at risk for acquired immune deficiency syndrome (AIDS). Rev Invest Clin 1983; $56: 126-9$.

2. Merk A, Subramaniam S. HIV-1 envelope glycoprotein structure. Struct Biol $2013 ; 23: 268-76$.

3. Xiang L, Lavanya Krishnana PC, Engelman A. Structural biology of retroviral DNA integration. Virology $2011 ; 411$ : 194-205.

4. Turner BG, Summers MF. Structural biology of HIV. J Mol Biol 1999 ; 285 : 1-32.

5. Briggs JA, Kräusslich HG. The molecular architecture of HIV.J Mol Biol 2011 ; 410 : 491-500.

6. Ganser BK. Assembly and analysis of conical models for the HIV-1 core. Science $1999 ; 283: 80-3$

7. Jouvenet N. La biogenèse du VIH-1 dévoilée. Med Sci (Paris) 2008 ; 11 : 908-10.

8. Lelek M, Nunzio F Di, Henriques R, et al. Superresolution imaging of HIV in infected cells with FIAsH-PALM. Proc Natl Acad Sci USA $2012 ; 109$ : 8564-9.

9. Kuznetsov YG, Victoria JG, Robinson WE, et al. Atomic force microscopy investigation of human immunodeficiency virus (HIV) and HIV-infected lymphocytes. J Virol 2003; 77 : 11896-909.

10. Baclayon M, Wuite GJL, Roos WH. Imaging and manipulation of single viruses by atomic force microscopy. Soft Matter $2010 ; 6: 5273-85$.

11. Nisole S, Saïb A. Early steps of retrovirus replicative cycle. Retrovirology $2004 ; 1: 9$.

12. Corbin A, Grigorov B, Roingeard P, et al. Une nouvelle vision de l'assemblage du VIH-1. Med Sci (Paris) $2008 ; 24: 49-55$.

13. Binnig G, Quate C, Gerber C. Atomic force microscope. Phys Rev Lett 1986 ; 56 : 930-3.

14. Radmacher M, Tillamnn RW, Fritz M, et al. From molecules to cells: imaging soft samples with the atomic force microscope. Science $1992 ; 257: 1900-5$.

15. Giocondi M, Milhiet PE, Lesniewska $\varepsilon$, et al. Microscopie à force atomique : de l'imagerie cellulaire à la manipulation moléculaire. Med Sci (Paris) $2003 ; 19$ : 92-9.

16. Parot $P$, Dufrêne $Y F$, Hinterdorfer $P$, et al. Past, present and future of atomic force microscopy in life sciences and medicine. J Mol Recognit 2007 ; 20 : 418-31.
17. Roos WH, Bruinsma R, Wuite GJL. Physical virology. Nat Phys 2010 ; 6 : 733-43.

18. Kol N, Shi Y, Tsvitov M, et al. A stiffness switch in human immunodeficiency virus. Biophys J $2007 ; 92: 1777-83$.

19. Heinz WF, Hoh JH. Spatially resolved force spectroscopy of biological surfaces using the atomic force microscope. Trends Biotechnol $1999 ; 17$ : 143-50.

20. Puchner $\varepsilon M$, Gaub HE. Force and function: probing proteins with AFM-based force spectroscopy. Curr Opin Struct Biol 2009 ; 19 : 605-14.

21. Bizzarri AR, Cannistraro $S$. The application of atomic force spectroscopy to the study of biological complexes undergoing a biorecognition process. Chem Soc Rev 2010 ; 39 : 734-49.

22. Dobrowsky TM, Rabi SA, Nedellec R, et al. Adhesion and fusion efficiencies of human immunodeficiency virus type l (HIV-1) surface proteins. Sci Rep $2013 ; 3: 3014$.

23. Faivre-Moskalenko C, Bernaud J, Thomas A, et al. RNA control of HIV-1 particle size polydispersity. PLoS One 2014 ; 9 : e83874.

24. Mateu MG. Mechanical properties of viruses analyzed by atomic force microscopy: a virological perspective. Virus Res $2012 ; 168: 1-22$.

25. Pang HB, Hevroni L, Kol N, et al. Virion stiffness regulates immature HIV-1 entry. Retrovirology $2013 ; 10: 4$.

26. Sieben C, Kappel C, Zhu R, et al. Influenza virus binds its host cell using multiple dynamic interactions. Proc Natl Acad Sci USA 2012 ; 109 : 13626-31.

27. Carrasco C, Douas M, Miranda R, et al. The capillarity of nanometric water menisci confined inside closed-geometry viral cages. Proc Natl Acad Sci USA $2009 ; 106: 5475-80$

28. Carrasco C, Castellanos M, de Pablo PJ, et al. Manipulation of the mechanical properties of a virus by protein engineering. Proc Natl Acad Sci USA $2008 ; 105: 4150-5$.

29. Plomp M, Rice MK, Wagner EK, et al. Rapid visualization at high resolution of pathogens by atomic force microscopy: structural studies of herpes simplex virus-1. Am J Pathol $2002 ; 160: 1959-66$.

30. Castellanos M, Pérez R, Carrillo PJP, et al. Mechanical disassembly of single virus particles reveals kinetic intermediates predicted by theory. Biophys J $2012 ; 102: 2615-24$

31. Ivanovska I, Wuite $G$, Jönsson $B$, et al. Internal DNA pressure modifies stability of WT phage. Proc Natl Acad Sci USA 2007 ; 104 : 9603-8.

32. Horowitz $\varepsilon D$, Rahman KS, Bower BD, et al. Biophysical and ultrastructural characterization of adeno-associated virus capsid uncoating and genome release. J Virol $2013 ; 87: 2994-3002$.

33. Kabla $A$, Chalut $K$. La réponse inhabituelle des noyaux de cellules souches embryonnaires aux forces mécaniques. Med Sci (Paris) 2014 ; 30 : 1061-3.

34. Muriaux D, Mirro J, Harvin D, Rein A. RNA is a structural element in retrovirus particles. Proc Natl Acad Sci USA 2001 ; 98 : 5246-51.

35. Kasas S, Stupar P, Longo G, Dietler G. Détecter la vie grâce à la microscopie à force atomique. Med Sci (Paris) $2015 ; 31: 369-71$.

\section{LA FONDATION PREMUP : UN OPÉRATEUR DE TERRAIN EN PÉRINATALITÉ RECONNU POUR SON EXCELLENCE ET SON INTERDISCIPLINARITÉ}

La Fondation de coopération scientifique PremUp, unique en Europe, intervient sur la prévention du handicap à la naissance, par la protection de la santé de la femme enceinte et du nouveau-né.

TIRÉS À PART

C. Moskalenko

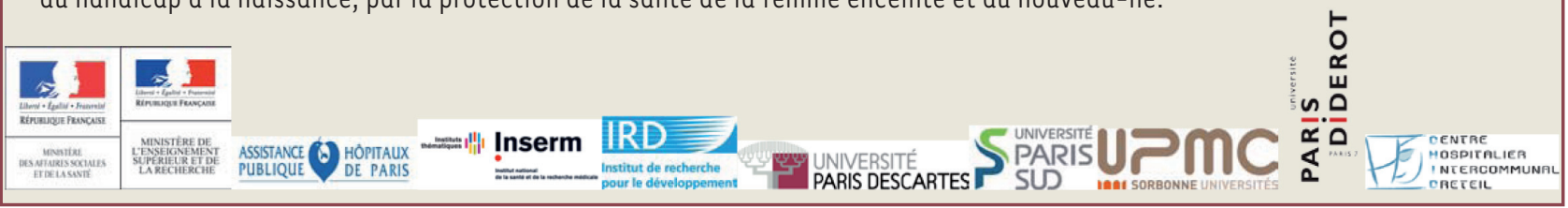

